\title{
Cáncer Orofaríngeo: Caracterización de la Mortalidad en Urbanización Valparaíso-Viña del Mar 2001 - 2010
}

\author{
Oropharyngeal Cancer: Characterization of Mortality in \\ the Urbanization Valparaíso-Viña del Mar 2001 - 2010
}

Carolina Colil Muñoz ; Alfredo Cueto Urbina*; Alejandra Fernández Moraga ${ }^{* * *}$ \& Alfredo Esguep Sarah ${ }^{* * *}$

COLIL, M. C.; CUETO, U. A.; FERNÁNDEZ, M. A. \& ESGUEP, S. A. Cáncer orofaríngeo: caracterización de la mortalidad en urbanización Valparaíso-Viña del Mar 2001 - 2010. Int. J. Odontostomat., 7(1):117-123, 2013.

RESUMEN: El cáncer orofaríngeo es un problema de salud de alcance mundial, según la OMS. Es el octavo mas frecuente a nivel mundial en población masculina. Se estima la incidencia anual en alrededor de 275.000 para cáncer bucal y 130.000 para cáncer faríngeo. Dos tercios de los casos ocurren en países en vías de desarrollo. En Chile el $1 \%$ del todos los cánceres corresponden a cáncer orofaríngeo, con tasa de mortalidad histórica de 1,3 x 100.000 hab. El propósito del estudio fue caracterizar la mortalidad por Cáncer Orofaríngeo en la urbanización Valparaíso-Viña del Mar, para la década 2001- 2010. Los datos de fallecidos fueron obtenidos de los libros de defunciones del archivo de las Oficinas del Registro civil de cada comuna. Las tasas de mortalidad fueron calculadas con datos poblaciones proporcionados por el Instituto Nacional de Estadística. La tasa de mortalidad para la conurbación Valparaíso-Viña del Mar alcanzo el 2,6 x 100.000 hab. Con una razón hombre: mujer de 1,3 a 1. La edad media de fallecimiento fue de 70,59 años. En cuanto a ubicación anatómica más frecuente fue orofaringe, luego glándulas salivales y lengua. Diagnóstico histológico más común fue Carcinoma Espinocelular y $24,8 \%$ de fallecidos presentó metástasis. Este estudio se concluye que existe un aumento de la tasa de mortalidad por Cáncer orofaríngeo (CFB) en las conurbación Valparaíso-Viña del Mar respecto a lo informado en el pasado para el país, esto podría estar demostrando un cambio de tendencia fundado en los cambios ambientales y conductuales de la población.

PALABRAS CLAVE: mortalidad por Cáncer, cáncer orofaríngeo, cáncer oral.

\section{INTRODUCCIÓN}

El cáncer orofaríngeo (COF) es un problema de salud de alcance mundial, según lo afirma la OMS. A escala mundial es el $8^{\circ}$ en frecuencia dentro de la población masculina (OMS, 2004), aunque según estudios de la Universidad de Barcelona en países menos industrializados es el 5to más frecuente en hombres y el 7 mo en mujeres (Sandoval, 2003). Se estima que la incidencia anual es de alrededor de 275.000 casos para cáncer bucal y de 130.000 afectados para cáncer en ubicación faríngea, de estos dos tercios de los casos ocurren en países en vías de desarrollo (Warnakulasuriya, 2009). En el año 2008 se registraron 128.000 muertes por esta causa (Jemal, 2011) y en general los hombres son más afectados que las mujeres (Warnakulasuriya).
El cáncer orofaríngeo más común histológicamente es el Carcinoma Espinocelular (Milán \& González, 1995). Presenta diferentes grados de diferenciación y predisposición a metastizar hacia los nódulos linfáticos. A nivel intraoral es más frecuente en el piso de boca luego lengua en su porción ventrolateral y paladar blando. Los pacientes que fallecen a causa de esta enfermedad lo hacen después de los 45 años, especialmente entre la $5^{\circ}$ y $6^{\circ}$ década de vida (Kumar et al., 2008). La tasa de supervivencia global es de un $50 \%$. El COF es responsable del $2 \%$ de los fallecimientos anuales en hombres y del $1 \%$ en las mujeres (Sapp et al., 2005).

En Chile el $1 \%$ del total de los cánceres co-

*Cirujano Dentista, Practica Privada, Viña del Mar, Chile.

"Cirujano Dentista, Profesor Titular Departamento de Salud Pública, Facultad de Odontología Universidad Andrés Bello, Viña del Mar, Chile.

"*'Cirujano Dentista, Docente Cátedra de Patología, Universidad Andrés Bello, Viña del Mar, Chile.

${ }^{* m+*}$ Profesor titular Departamento de Cirugía y Patología, Facultad de Odontología, Universidad Andrés Bello, Viña del Mar, Chile. 
rresponden a cáncer orofaríngeo, con una tasa de mortalidad histórica de 1,3 x 100.000 hab. Es más frecuente en hombres que en mujeres en una proporción de 2,3:1 y en mayores de 45 años (Riera \& Martínez, 2005).

Siendo el cáncer en general, responsable de una alta y creciente morbilidad y mortalidad en las sociedades modernas se considera un problema de salud pública, por ello es relevante su estudio según las diferentes zonas anatómicas que afecta en especial el cáncer orofaríngeo que puede estar sufriendo modificaciones por los cambios tanto demográficos, ambientales como de estilo de vida en los que la población está inmersa.

Esta investigación tiene como objetivo caracterizar la mortalidad por Cáncer Orofaríngeo en las ciudades de urbanización Valparaíso-Viña del Mar, para la década 2001- 2010.

\section{MATERIAL Y MÉTODO}

Se realizó un estudio de corte transversal, la población estudiada correspondió a todos los fallecidos por cáncer con localización anatómica dentro del territorio buco faríngeo que incluye la boca, orofaringe, nasofaringe y piel de la zona tanto de tejidos blandos como duros, de origen primario o por metástasis, con causa de muerte mediata o inmediata, para el periodo enero 2001 a diciembre 2010, urbanización Valparaíso-Viña del Mar.

Se recogieron los datos a través de un censo que se realizó a partir de los certificados de defunción, los cuales registran el detalle de todas las muertes que se produjeron en la urbanización Valparaíso-Viña del Mar. Estos certificados se encuentran en la sección de archivo, subsección de defunciones en las oficinas de Registros Civil de cada comuna. Los datos recogidos se ingresaron a una base que incluía identificación del fallecido, edad al momento del fallecimiento, género, lugar de fallecimiento, causa de muerte mediata y causa de muerte inmediata, diagnóstico histológico en detalle y simplificado, ubicación o localización anatómica de la lesión, presencia de metástasis según consignó el médico que constató la muerte de cada paciente. Para determinar el diagnóstico de los tumores se uso la clasificación WHO modificada (Barnes et al., 2005).

Para el cálculo de las tasas de mortalidad, en su denominador se ingresaron los datos poblacionales proporcionados por el Informe de Demografía que publica el Instituto Nacional de Estadística que entrega la población estimada por comuna al 30 de junio de cada año, que incluye además la distribución de la población por, sexo y grupos etarios.

El análisis estadístico al cual se sometieron los datos fue en un primer nivel de carácter descriptivo para las variables cualitativas y cuantitativa, en las primeras se calcularon frecuencias y porcentaje; para las segundas se calcularon medias, modas, medianas y desviación estándar. Para el análisis bivariante se calculó la significación estadísticamente con la aplicación del test correspondiente en el caso de relaciones entre variables cualitativas el Chi cuadrado y entre una variable cualitativa y una cuantitativa múltiple se aplicó el ANOVA. Los resultados se consideraron estadísticamente significativos con un nivel de error de 5\%. Para los cálculos se utilizó el software SPSS versión 19 y Microsoft Office Excel 2007.

\section{RESULTADOS}

El total de fallecidos de las ciudades de urbanización Valparaíso-Viña del Mar ascendió a 51.034 para la década 2001 - 2010 de los cuales 10.313 (20,2\%) fallecieron por cualquier tipo de cáncer, entre estos 145 $(0,28 \%$ del total de fallecidos) fallecieron por cáncer orofaríngeo lo que representa el 1,4\% de todos los cánceres, detalle por comuna se observa en la Tabla I.

De estos pacientes el 64,8\% muere directamente por causa inmediata del COF, el restante $35,2 \%$ el cáncer esta consignado como causa mediata pues mueren por complicaciones al sistema cardiovascular $(13,8 \%)$, o sistema respiratorio $(13,1 \%)$, o falla multisistémica $(6,9 \%)$ o causa infecciosa $(1,4 \%)$. Del total de los casos $69 \%$ fallecieron en su domicilio y el restante $31 \%$ en institución hospitalaria tanto públicas como privadas.

La media de la tasa de mortalidad específica para la década en estudio alcanzó los 2,6 por 100.000 hab. mostrando diferencias según género, así los hombres tuvieron una tasa de 2,9 por 100.000 hab., en cambio esta tasa en mujeres se reduce a 2,2 por 100.000 hab. Se presentaron 81 casos entre los hombres y 64 casos entre las mujeres dando una razón de 1,3 hombres por 1 mujer. El comportamiento de las tasas de mortalidad específicas de COF por año se observan en la Tabla II. 
COLIL, M. C.; CUETO, U. A.; FERNÁNDEZ, M. A. \& ESGUEP, S. A. Cáncer orofaríngeo: caracterización de la mortalidad en urbanización Valparaíso-Viña del Mar 2001 - 2010. Int. J. Odontostomat., 7(1):117-123, 2013

Tabla I. Frecuencia de defunciones en general, por cáncer y por cáncer orofaríngeo. Década 2001 - 2010 Valparaíso Viña del Mar.

\begin{tabular}{lcccc}
\hline & \multicolumn{2}{c}{ Valparaíso } & \multicolumn{2}{c}{ Viña del Mar } \\
\cline { 2 - 5 } Comuna & Frecuencia & Porcentaje & Frecuencia & Porcentaje \\
\hline Frecuencia de fallecid os por comuna & 25853 & $100 \%$ & 25181 & $100 \%$ \\
Frecuencia de fallecid os por cáncer & 4282 & $16,56 \%$ & 6031 & $23,95 \%$ \\
Frecuencia de fallecid os por cáncer & 77 & $0,30 \%$ & 68 & $0,27 \%$ \\
Proporción Cáncer Bucofaríngeo/Total de & \multicolumn{2}{c}{$1,8 \%$} & $1,1 \%$ \\
\hline
\end{tabular}

Tabla II. Tasa de mortalidad general y por sexo de cáncer orofaríngeo. Periodo 2001 a 2010 Fusión Comuna de Valparaíso y Viña del Mar.

\begin{tabular}{|c|c|c|c|c|c|c|}
\hline \multirow[b]{2}{*}{ Año } & \multicolumn{2}{|c|}{ Frecuencia } & \multirow{2}{*}{$\begin{array}{c}\text { Total Casos } \\
\text { CBF }\end{array}$} & \multicolumn{2}{|c|}{ Tasa por sexo } & \multirow{2}{*}{$\begin{array}{c}\text { Tasa } \\
\text { Específica }\end{array}$} \\
\hline & Hombre & Mujer & & Hombre & Mujer & \\
\hline 2001 & 8 & 4 & 12 & 2,89 & 1,37 & 2,11 \\
\hline 2002 & 8 & 4 & 12 & 2,89 & 1,37 & 2,11 \\
\hline 2003 & 7 & 5 & 12 & 2,53 & 1,71 & 2,11 \\
\hline 2004 & 19 & 6 & 26 & 6,86 & 2,05 & 4,56 \\
\hline 2005 & 10 & 4 & 13 & 3,61 & 1,37 & 2,28 \\
\hline 2006 & 4 & 6 & 10 & 1,44 & 2,06 & 1,76 \\
\hline 2007 & 5 & 17 & 22 & 1,81 & 5,84 & 3,88 \\
\hline 2008 & 8 & 5 & 13 & 2,90 & 1,72 & 2,30 \\
\hline 2009 & 9 & 6 & 15 & 3,27 & 2,07 & 2,65 \\
\hline 2010 & 3 & 7 & 10 & 1,09 & 2,42 & 1,77 \\
\hline Total & 81 & 64 & 145 & 2,93 & 2,20 & 2,55 \\
\hline
\end{tabular}

Tabla III. Edad de fallecimiento por cáncer orofaríngeo según sexo.

\begin{tabular}{llccc}
\hline Distribución por sexo & & Hombre & Mujer & Ambos \\
\hline Media & & 66,38 & 75,91 & 70,59 \\
Intervalo de confianza & Límite inferior & 62,87 & 72,47 & 68,01 \\
para la media al 95\% & Límite superior & 69,90 & 79,35 & 73,16 \\
Media recortada al 5\% & 67,33 & 77,30 & 71,81 \\
Mediana & 70,00 & 79,00 & 73,00 \\
Varianza & 252,41 & 189,58 & 245,69 \\
Desv. típ. & 15,89 & 13,77 & 15,67 \\
Mínimo & 18 & 25 & 18 \\
Máximo & 96 & 94 & 96 \\
Rango & 78 & 69 & 78 \\
Amplitud intercuartil & 17 & 14 & 20 \\
Asimetría &,- 991 & $-1,694$ & $-1,171$ \\
Curtosis & 1,664 & 3,446 & 1,721
\end{tabular}

La estadística descriptiva de la edad al momento del fallecimiento por COF se informa en la Tabla III, en ella se constatan las diferencias estadísticamente sig- nificativas ( $p$-valor 0,000 ) según género, siendo los hombres los que mueren en forma mas prematura que las mujeres. 
Tabla IV. Edad de defunción según el tipo de cáncer orofaríngeo.

\begin{tabular}{lccccc}
\hline & & & & \multicolumn{2}{c}{ Intervalo de confianza para la media al } \\
\cline { 3 - 6 } & $\mathbf{n}$ & Media & Desv. Típ. & Límite inferior & Límite superior \\
\cline { 2 - 6 } Carcinoma & 96 & 70,31 & 15,41 & 67,19 & 73,44 \\
Linfoma & 7 & 75,57 & 8,56 & 67,65 & 83,49 \\
Melanoma & 4 & 84,50 & 9,68 & 69,10 & 99,90 \\
Osteosarcoma & 4 & 47,00 & 29,65 & $-0,19$ & 94,19 \\
Total & 111 & 70,32 & 16,26 & 67,26 & 73,37 \\
\hline
\end{tabular}

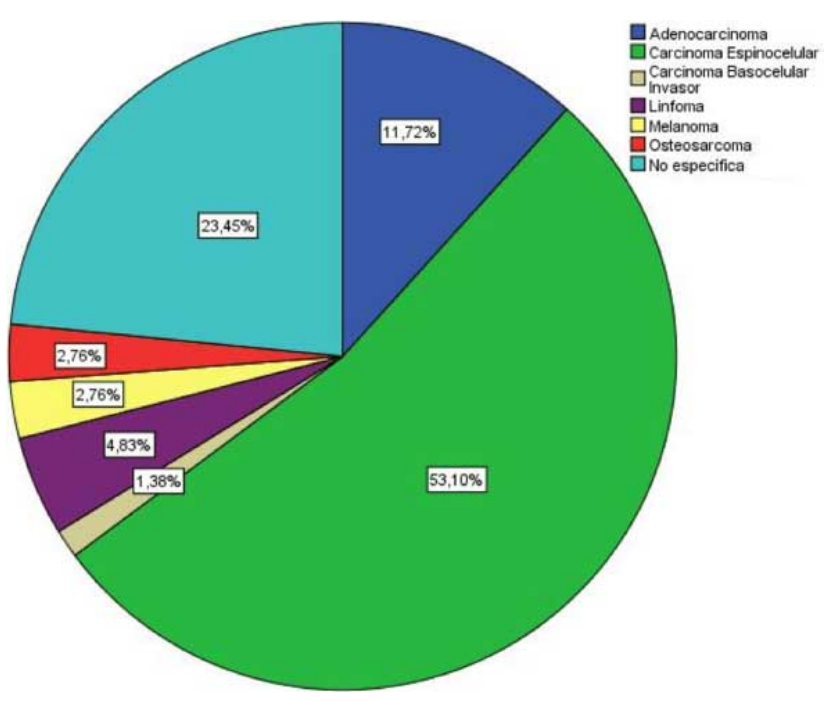

La distribución del diagnóstico histológico del COF se detalla en la Figura 1, donde el Cáncer Espino Celular es el principal tipo histológico diagnosticado.

Los resultados de la media de la edad de fallecimiento según el tipo histológico simplificado de COF (Tabla IV), informa diferencias significativas entre Osteosarcoma y el resto de los tipo de cánceres que incluye los tipo Carcinoma, Linfoma, Melanoma (p-valor 0,006).

La distribución del COF según ubicación anatómica se observa en la Figura 2 ordenados según frecuencia y porcentaje de participación en el total. La razón hombre: mujer para las diferentes localizaciones fueron Orofaringe 1,4:1, Glándulas Salivales

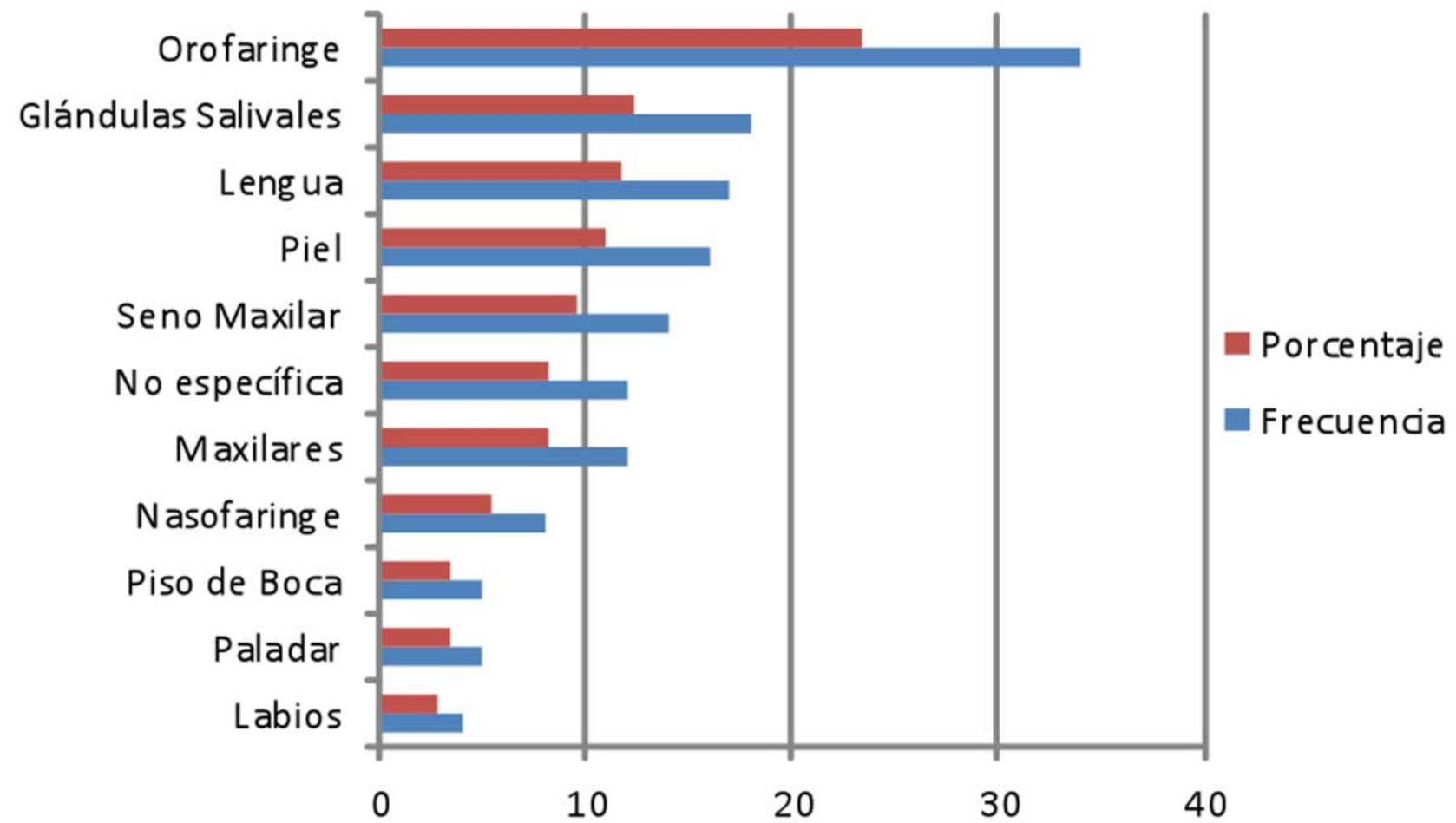

Fig. 2. Distribución del cáncer orofaríngeo por ubicación en frecuencia y porcentaje. 
0,8:1, Lengua 1,8: 1, Piel 1,7:1, Seno Maxilar 0,8: 1, Huesos maxilares 1:1, Nasofaringe 0,6:1, Piso de Boca 4:1, Paladar 4:1 y Labios 3:1, con un solo caso para las mujeres en cada una de los tres últimos sitios anatómicos.

De los cánceres estudiados presentaron metástasis el $24,8 \%$, siendo la mas frecuente la metástasis de ubicación múltiple y luego la cervical como se observa en la Tabla V.

Tabla V. Localización de metástasis de cáncer orofaríngeo.

\begin{tabular}{lcc}
\hline & Frecuencia & Porcentaje \\
\hline Sin metástasis & 109 & 75,2 \\
Con metástasis & & \\
Múltiples & 10 & 6,9 \\
Cervical & 4 & 2,8 \\
Cerebrales & 2 & 1,4 \\
Hepáticas & 2 & 1,4 \\
Pulmonares & 1 & 0,7 \\
Seno Maxilar & 1 & 0,7 \\
No especifica & 16 & 11,0 \\
Total & 145 & 100,0 \\
\hline
\end{tabular}

\section{DISCUSIÓN}

El principal hallazgo de esta investigación son los altos niveles de tasa de mortalidad específica por cáncer orofaríngeo en urbanización Valparaíso-Viña del Mar para la década 2001 a 2010, de hecho la media de esta tasa de mortalidad alcanzó 2,6 por 100.000 habitantes y los casos de COF representaron el $1,4 \%$ de todos los cánceres. Ambas cifras preocupantes pues si se compara con Chile para el periodo 1955 a 2002 se informaron tasas que fluctuaban de 0,9 a 1,3 por 100.000 habitantes y que los casos durante ese periodo no superaban $1 \%$ de todos los cánceres (Riera \& Martínez).

Otro hallazgo relevante fue que las tasas según género son mayores para los hombres pero acercándose a las mujeres, los primeros tasa de 2,9 y las segundas tasa de 2,2 ambas por 100.000 habitantes. Ambas además resultan mayores que las informadas en otros estudios a nivel nacional (Riera \& Martínez) y diferentes a nivel internacional así tenemos en Argentina en la provincia de Córdova para el periodo 1997-2000 (Morelatto \& López de Blanc, 2006), se informó de tasa de 1,6 para hombres de 0,4 para mujeres por cada 100.000 habitantes. Y en Cuba para el periodo de 1987 a 1996 la población masculina tiene mayores tasas de mortalidad (entre 5,48 y 6,79 ) en comparación con las mujeres (entre 1,37 y 1,83 ). Sin embargo. la comparación internacional se hace compleja por los diferentes sistemas de medición y diferentes factores de riesgo a que esta sometida cada población, así en Cuba se podría explicar ese aumento por el tipo de uso que le da la población al tabaco y por su fácil acceso (García et al., 1999).

La alta tasa de mortalidad por COF de la urbanización Valparaíso-Viña del Mar se podría explicar por las variaciones en las costumbres o estilos de vida entre otros por los hábitos alimenticios, las modificaciones en el medio ambiente lo que ha incidido en cambio en la manufactura y origen de los alimentos, además la cuidad de Valparaíso concentra población mas envejecida que el resto del país. Por otro lado es conocida la relación causal entre tabaco y cáncer, de hecho este incremento de tasa de mortalidad para la zona en gran parte se podría entender por el aumento de la costumbre de fumar tabaco en Chile, la tendencia a igualar este hábito entre hombres y mujeres, incluso estas últimas entre los mayores de 65 años son las que mas fuman y la región de Valparaíso concentra una alta tasas de prevalencia del hábito tabáquico en comparación al resto del país (MINSAL, 2010).

En consecuencia a futuro se podría esperar que siguiera incrementándose los casos en esta zona y situación similar podría ocurrir en el resto del país, por ello los programas de salud que consideran el consejo antitabaco entregado por el odontólogo a sus pacientes impulsados por el Ministerio de Salud debería mantenerse y perfeccionarse, pues contribuiría a que la población accediera a mayor grado de conocimientos de los riesgos del tabaco y por otro lado que el odontólogo general realizara actividades de prevención, de diagnóstico precoz de los casos y practicara la oportuna derivación (MINSAL, 2011).

Este estudio se realizó sobre registros de alta confiabilidad pues cada certificado revisado fue emitido por un médico cirujano que atestiguaba la causa de muerte inmediata y mediata. Además a partir de la información que maneja el registro civil que es reconocido por su exactitud de datos se nos validaba la fecha de nacimiento, la fecha de defunción, el gé- 
nero del fallecido, el lugar del fallecimiento. Por otro lado las tasas en sus denominadores se basaron en poblaciones proporcionadas por los Informes Demográficos confeccionados por el reconocido Instituto Nacional de Estadísticas. Y los análisis estadísticos a los cuales fueron sometidos los datos corresponden según el comportamiento y tipos de variables. Las ciudades de urbanización Valparaíso-Viña del Mar concentran una importante población urbana del país del orden del $4,2 \%$. En consecuencia esta zona podría ser considerada adecuada para representar al Chile urbano, por tanto este censo local podría tomarse como una muestra nacional este es el fundamento para hacer inferencia de los resultados locales.

El fallecimiento por diagnostico de COF en nuestra muestra afectó desde los 18 a 96 años, pero la media fue de 70,59 años (DE 15,67) y la edad de fallecimiento fue significativamente diferente entre hombres (66,38 años) y mujeres (75,91 años), los primeros mueren prematuramente 11 años antes que las mujeres, es probable que se deba a la exposición más precoz de los hombres a los factores de riesgo entre estos a tabaco y alcohol.

Según las edades de fallecimiento de los casos de nuestro estudio, se observa que esta es una enfermedad que afecta principálmente a la tercera edad, coincidiendo con lo reportado en Chile y Argentina por otros autores (Riera \& Martínez; Morelatto \& López de Blanc). En esa etapa de la vida pueden estar ocurriendo alteraciones en la regularización del ciclo celular que conducen a una acumulación de daño genético (Bettendorf et al., 2004).

De acuerdo a los sitios mas afectados por COF se encuentra la orofaringe lo que coincide con estudio de Sánchez et al. (2003). Si solo se considera el cáncer bucal u oral los sitios más afectados en nuestro estudio fueron glándulas salivales y lengua. La razón hombre mujer para los diferentes localizaciones de cáncer en nuestro estudio demuestra que afecta mas el cáncer de lengua, piel y orofaringe a los hombres. Se podría explicar estos resultados debido a que los hombres consultan menos al odontólogo que las mujeres y por otra parte el examen oral en ocasiones no incorpora la revisión de los bordes laterales, ni del vientre lingual.

La razón hombre mujer en general por COF está mas cercana en este estudio que lo informado anteriormente por otras investigaciones, lo que estaría ocurriendo es que tanto los hombres como las mujeres están compartiendo los mismos factores de riesgo, situación que no ocurría en el pasado.

El principal diagnóstico histológico que se reportó en los 111 casos fue el Carcinoma Espinocelular $(53,1 \%)$, luego Adenocarcinoma $(11,7 \%)$. Tanto el sitio y diagnóstico histológico más frecuente coinciden con lo reportado a nivel nacional (Riera \& Martínez).

Nuestro estudio de acuerdo a nuestra búsqueda parece ser el primero que reporte sobre presencia de metástasis y ubicación de está en COF. Del total de casos $24,6 \%$ se diagnosticó con metástasis siendo la mas frecuente las múltiples y cervicales, estas últimas se podrían explicar por la vecindad del territorio afectado inicialmente.

Una limitación del estudio fue que no permitió hacer estimaciones de futuro confiables, ya que el periodo recogido de una década es breve para hacer proyecciones. Una debilidad adicional es que en unas variables para algunos casos no presentaban datos pues no lo contenía el certificado de defunción, así tenemos sin datos del sitio específico que afectaba el cáncer (12 casos, $8,3 \%$ ), ni presencia de metástasis (16 casos $11 \%$ ) ni del diagnóstico histológico (34 casos, 23,4\%), es posible que en algunos de esos casos jamás se diagnosticó el tipo de histológico ni fue relevante la ubicación original por el estado de avance que se presentaba la patología al momento de requerir asistencia médica. A pesar de ello las tendencias en estas variables no fueron modificadas por estos casos perdidos pues se encontró coincidencia con otros reportes.

Este estudio concluye que existe un aumento de la tasa de mortalidad por COF en las ciudades de urbanización Valparaíso-Viña del Mar respecto a lo informado en el pasado para el país, esto podría estar demostrando un cambio de tendencia fundado en los cambios ambientales y conductuales de la población. El COF afecta mas en la tercera edad, es mas frecuente en hombres, pero se están acercando la razón hombre: mujer, sus localizaciones principales son lengua, glándulas salivales, orofaringe, puede dar metástasis y el diagnóstico histológico más común es Carcinoma Espinocelular.

Estas orientaciones diagnósticas se hacen necesario que las maneje el odontólogo y el médico general para mejorar el diagnóstico precoz y la derivación oportuna al patólogo y oncólogo según corresponda. 
COLIL, M. C.; CUETO, U. A.; FERNÁNDEZ, M. A. \& ESGUEP, S. A. Oropharyngeal cancer: characterization of mortality in the urbanization Valparaíso-Viña del Mar 2001 - 2010. Int. J. Odontostomat., 7(1):117-123, 2013.

SUMMARY: The oropharyngeal cancer is a worldwide health problem according to WHO. It is the eighth most common in the world male population. Annual incidence is estimated at around 275,000 for oral cancer 130,000 and for pharyngeal cancer. Two thirds of the cases occur in developing countries. In Chile $1 \%$ of all cancers are oropharyngeal cancer, with historical mortality rate of 1.3 per 100,000 inhabitants. The purpose of the study was to characterize oropharyngeal cancer mortality in the urbanization Valparaíso-Viña del Mar, for the decade 2001-2010. Deaths data were obtained from books File deaths of civil registry offices of each commune. Mortality rates were calculated using population data provided by the National Statistics Institute. The mortality rate for the conurbation Valparaíso-Viña del Mar reached 2.6 per 100,000 inhabitants. With a male: female ratio of 1.3 to 1 . The average age of death was 70.59 years. As most frequent anatomical location oropharynx, salivary glands and tongue then. The most common histologic diagnosis was Squamous Cell Carcinoma and $24.8 \%$ of deaths presented metastasis. This study concludes that there is an increase in the rate of oropharyngeal cancer mortality in the conurbation Valparaíso-Viña del Mar about what was reported in the past for the country, this could be showing a trend change based on environmental and behavioral changes of the population.

KEYWORDS: cancer mortality, oropharyngeal cancer, oral cancer.

\section{REFERENCIAS BIBLIOGRÁFICAS}

Barnes, L.; Eveson, J. W.; Reichart, P. \& Sidransky, D. World Health Organization Classification of Tumours. Pathology and Genetics of Head and Neck Tumours. Lyon, France, IARC Press, 2005.

Bettendorf, O.; Piffko, J. \& Bánkfalvi, A. Prognostic and predictive factors in oral squamous cell cancer: important tools for planning individual therapy? Oral Oncol., 40(2):110-9, 2004.

García, M.; Lence, J.; Sosa, M.; Martín, L. \& Fernández, L. Mortalidad del cáncer bucal en Cuba (1987-1996). Rev. Cubana Oncol., 15(2):114-8, 1999.

Jemal, A.; Bray F.; Center, M. M.; Ferlay, J.; Ward, E. \& Forman, D. Global cancer statistics. CA Cancer J. Clin., 61(2):69-90, 2011

Kumar, S. K.; Shuler, C. F.; Sedghizadeh, P. P. \& KalMar J. R. Oral mucosal melanoma with unusual clinicopathologic features. J. Cutan. Pathol., 35(4):392-7, 2008.

Milán, A. \& González, M. Tumores malignos de la mucosa oral. En: Bagán, J. V. (Ed). Medicina Oral. Barcelona, Masson, 1995.

Ministerio de Salud (MINSAL). Encuesta Nacional de Salud 2009-2010. Santiago, Ministerio de Salud, Gobierno de Chile, 2010. Disponible en: http://www.redsalud.gov.cl/portal/url/item/99bbf09a908d3eb8e04001011f014b49.pdf

Ministerio de Salud (MINSAL). Prevención del consumo del tabaco. Santiago, Ministerio de Salud, Gobierno de Chile, 2011. Disponible en: http://www.minsal.gob.cl/portal/url/ page/minsalcl/g_proteccion/g_tabaco/prev_tabaco.html

Morelatto, R. A. \& López de Blanc, S. A. Oral cancer mortality in the province of Cordoba, Argentine Republic in the period 1975-2000. A comparative study with other populations. Med. Oral Patol. Oral Cir. Bucal, 11(3):E230-5, 2006.
Organización Mundial de la Salud (OMS). Informe sobre el problema mundial de las enfermedades bucodentales. Organización Mundial de la Salud, 2004. Disponible en: http://www.who.int/mediacentre/news/releases/2004/pr15/ es/

Riera, P. \& Martínez, B. Morbilidad y Mortalidad por cáncer bucofaríngeo y faríngeo en Chile. Rev. Med. Chil., 133(5):555-63, 2005.

Sánchez, M. J.; Martínez, C.; Nieto, A.; Castellsagué, X.; Quintana, M. J.; Bosch, F. X.; et al. Oral and oropharyngeal cancer in Spain: influence of dietary patterns. Eur. J. Cancer Prev., 12(1):49-56, 2003.

Sandoval, M. Papel pronóstico de los factores clínicos y epidemiológicos en una cohorte de pacientes con cáncer de cavidad oral y orofaringea. Tesis de licenciatura para obtener la especialidad de Otorrinolaringología. Barcelona, Facultad de Medicina, Universidad de Barcelona, 2003.

Sapp, J.; Eversole, L. \& Wysocki, G. Patologia Oral y Maxilofacial contemporánea. 2a ed. Madrid. Elsevier, 2005.

Warnakulasuriya, S. Global epidemiology of oral and oropharyngeal cancer. Oral Oncol., 45(4-5):309-16, 2009.

Dirección para Correspondencia:

Alfredo Esguep Sarah

Departamento de Salud Pública

Facultad de Odontología

Universidad Andrés Bello

Avenida Valparaíso 1560

Viña del Mar

CHILE

Email: aesguep@unab.cl
Recibido : 01-09-2012

Aceptado: 03-12-2012 